\title{
The Helium Cryogenic System for the ATLAS Experiment
}

\author{
N. Delruelle, F. Hang, G. Passardi, H. Ten Kate
}

\begin{abstract}
Alystract-uThe magnetic configuration of the ATLAS detector is generated by an inmer superconducting solenoid and three air-core toroids (the barrel and twe end-caps), each of them made of eight superconducting coils, Two separated helium refrigerators will be used to altow conl-down from ambient temperature and steady-state operation at $4.5 \mathrm{~K}$ of all the magnets having a total cold mass of about 600 tons. In comparison with the preliminary design, the helium distribution scheme and interface with the magnet sub-systems are simplified, resulting in a consitlerable improvement of the operational easiness and the overall reliability of the system at some expense of the operational flexibility. The paper presents the cryogenic layout and the basic principles for magnets cool. down, steady state oporation and thermal recovery after a fast energy dump.
\end{abstract}

Index Terms-- Superconducting magnets, helium refrigeration

\section{INTRODUCTION}

The ATLAS collaboration is constructing a general-purpose detector for proton-proton collisions at an energy lovel of 14 Tev provided by the future Large Hadron Collider (LHC) at CERN.

The magnetic field configuration of the detector allows momentum measurements of the charged particles produced in protons collisions. Four superconducting magnets are needed, namely the Batrel Toroid (BT), the two End-Cap Toroids (ECT) and the Central Solenoid (CS). The CS, genorating the field for the inner detector, will be sturrounded by the three large air-core toroids (the BT and the two ECTs) generating the magnetic field for the muon

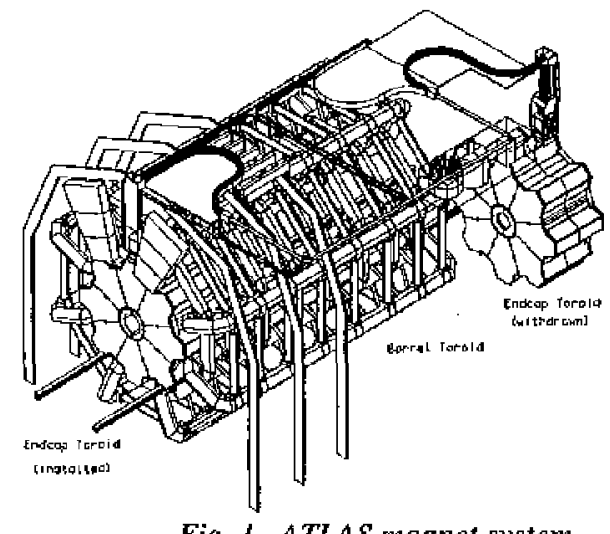

Fig. 1. ATLAS magnet system speciroineter [1][2].

The overall dimension of this magnet system (Fig, 1) extends over a length of $26 \mathrm{~m}$, an outer diameter of $20 \mathrm{~m}$, and with a total volume of $81000 \mathrm{~m}^{3}$. The magnets have a total stored energy of $1530 \mathrm{MJ}$, a total weight of more than 1'300 tons of which the cold mass represents about 600 tons. All magnets operate at a $4.5 \mathrm{~K}-4.8 \mathrm{~K}$ temperature level.

From a functional point of view, the cryogenic system for each superconducting magnet has been sub-divided into internal, proximity and external cryogenics. The cooling system of the superconducting coils and the thermal shields with their corresponding piping and feedthroughs represent the internal cryogenics. The auxiliary cryogenic equipment necessary for the opcration of each magtet like current leads, helium phase separators, buffer Dewars, liquid helium pumps, helium distribution valves and safety devices are called the proximity cryogenics. All these equipment installed close to the detector make the interface with the external cryogenics consisting of the helium refrigeration plants which are common to all magnets.

The thermal loads of the four magnets as well as the external cryogenics are summarized in table 1 .

To satisfy these thermal loads requirements, it is planned to re-use an existing $6 \mathrm{~kW} @ 4,5 \mathrm{~K}$ equivalent refrigerator. However, this cryoplant would have been insufficicnt to achieve a reasonable cool-down time of the magnets cold mass and to cope with the non-isothermal loads ( $40 \mathrm{~K}$ to $80 \mathrm{~K}$ ) of the thermal shiclds [3]. Therefore, it has been decided to use two separate units: the $6 \mathrm{~kW}$ plant plus a now refrigerator. The existing one will be used for the steady-state operation at $4.5 \mathrm{~K}$ and the cooling of the current loads. It will also allow the cool-down from $100 \mathrm{~K}$ as well as the thermal recovery after a fast onergy dump. The additional refrigerator will be solely dedicated to the operation of the themal shields and to the cool-down of magnets from ambient temperature to $100 \mathrm{~K}$, by making use of liquid nitrogen supplied from the surface.

\section{HELIUM DISTRHBUTION SYSTEM}

In the earlier design of the helium distribution. system [2][4], there were threc almost independent proximity cryogenic sub-systems: one for the BT [5], one for the two ECTs [6] and one for the CS [7]. Each of them was linked to the refrigerators via six helium lines. This six-line 


\begin{tabular}{|c|c|c|c|c|c|c|}
\hline \multicolumn{2}{|l|}{ Load type } & BT & Two ECTs & $\mathrm{CS}$ & Ptoximity \& & Total \\
\hline Stattic isothermal lond at $4.5 \mathrm{~K}$ & [W] & 660 & 360 & 80 & 1100 & 2200 \\
\hline Dynamic isothermal load at $4.5 \mathrm{~K}$ & [W] & 100 & 220 & 80 & - & 400 \\
\hline Liquefaction load at $4.5 \mathrm{~K}[\mathrm{~g} / \mathrm{s}]$ & & 3.3 & 6.5 & 1.2 & - & 11.0 \\
\hline Non-isothermal load between $40 \mathrm{~K}$ & and $80 \mathrm{~K}[\mathrm{~W}]$ & 6600 & $4 \overline{500}$ & 500 & 1900 & 13500 \\
\hline
\end{tabular}

Table 1; thermal loads of ATLAS nagnets

concept was studied to allow a high degree of operational flexibility for sequential cooling of all magnets from any temperature between a!nbient and $4.5 \mathrm{~K}$. For instance, some magnet(s) could have been in cool-down or in quench recovery mode, while the others remained in normal operation at $4.5 \mathrm{~K}$.

More recently, several simplifications have been introduced leading to a new distribution system (Fig. 2). The former three sub-systems ate grouped into two: one for all the toroids presently under study and one for the CS alreacly in the construction phase. Furthermore, in the new design, all the toroids are considered as a single cold mass allowing the reduction of the intercotnecting helium lines from six to four. In addition to an important cost saving, a considerable improvement of both the operational easiness and the overall reliability of the system were achieved, at some expense of the operational flexibility.

\section{BASIC OPERATIION PRTNCIPLES}

Wo have distinguished four nain operational modes: cooldown, warm-up, steady-state operation and thermal recovery after a fast energy dump.

The normal cool-down procedure foresces all magnets to be cooled down in parallel with a forced flow of high-pressure gaseous helium. In order to avoid excessive thermal stresses by a large temperature gradient in the cold mass, the magnets should be cooled by flowing gaz with a temperature maintained constantly $40 \mathrm{~K}$ lower than the temperature of the coils. From $300 \mathrm{~K}$ to $100 \mathrm{~K}$, coils and shields will be cooled down with helium provided by the additional refrigerator boosted by liquid nitrogen, while the $6 \mathrm{~kW}$ plant will take over the cool-down from $100 \mathrm{~K}$ to $4.5 \mathrm{~K}$, supplying directly into the coils supercritical helium at 3 bar and $4.5 \mathrm{~K}$. The total cool-down time will not exceed 40 days. Since the CS cold mass represents only $1 \%$ of the total cold mass and, therefore, needs a small fraction of the refrigerators capacity, cooldown from ambient temperature of the CS with the toroids in operation at $4.5 \mathrm{~K}$ still remains feasible.

On the contrary, the cooling-down of the toroids requires a temporary stop of the $4.5 \mathrm{~K}$ operation of the CS, since, with the new simpliffed distribution system, this sequential cooling is no longer possible.

The warm-up phase of all toroids will be done in the opposite way of the cool-down, by means again of forced flow of high pressure gaseous helium provided by the new refrigerntor, maintaining this time the helium temperature constantly

$40 \mathrm{~K}$ higher than the coils. Whereas for the CS, it has been preferted to warm it up by direct electrical heating through the windings.

For stcady-state operation, the refrigerator will supply supercritical helium at 3 bar and $4.5 \mathrm{~K}$ to bolh proximity sub-systems where the helium will be expanded to 1,3 bar. Although all magnets will be cooled indirectly by twophase helium flowing into pipes welded to the cold mass, different cooling priticiples have been adopted for the CS and for the toroids (BT and ECl's). Centrifugal pumps providing in total $1200 \mathrm{~g} / \mathrm{s}$ of saturated helium flow will be requested to keep the toroids at their operating temperature of $4.5 \mathrm{~K}$. This large flow should ensure the stability in the two-phase helium obtained by combining a mass flow rate per unit arca above $4 \mathrm{~g} \cdot \mathrm{s}^{-1} \cdot \mathrm{cm}^{-2}$ with a vapour mass fraction at the outlet of the coils less than 10\% [8][9]. For the CS having a more simple cylindrical structure and piping distribution, both two-phase heliurn forced flow provided by the refrigerator and thermosyphon cooling as back up mode are foreseen [10].

For the thermal recovery after a fast energy dump, two different scenarios can occur. If the CS quenches alonc, the BT and ECTs can be kept running at $4.5 \mathrm{~K}$ while the refrigerator recovers smoothly the operating conditions for the CS. If the toroids quench, their thermal recovery from the after-quench temperature $(\approx 60 \mathrm{~K})$ requires a temporary stop of the CS cooling. The quench recovery time will be less than 3 clays.

Finally, in order to avoid a fast energy dump of the coils in the case of a cryogenic plant failure, each proximity subsystem is equipped with a buffer Dewar which holds the required roserve volurno of liguid heliunt to allow a slow energy dump which lasts two hours.

\section{Iv. CONCLUSIONS}

The external helium cryogenic system for the ATLAS experiment has been finalized and is ready for the tendering of the different equipment. In comparison with the preliminary design, considerable simplifications have been introducesl. The former three proximity sub-systems, 


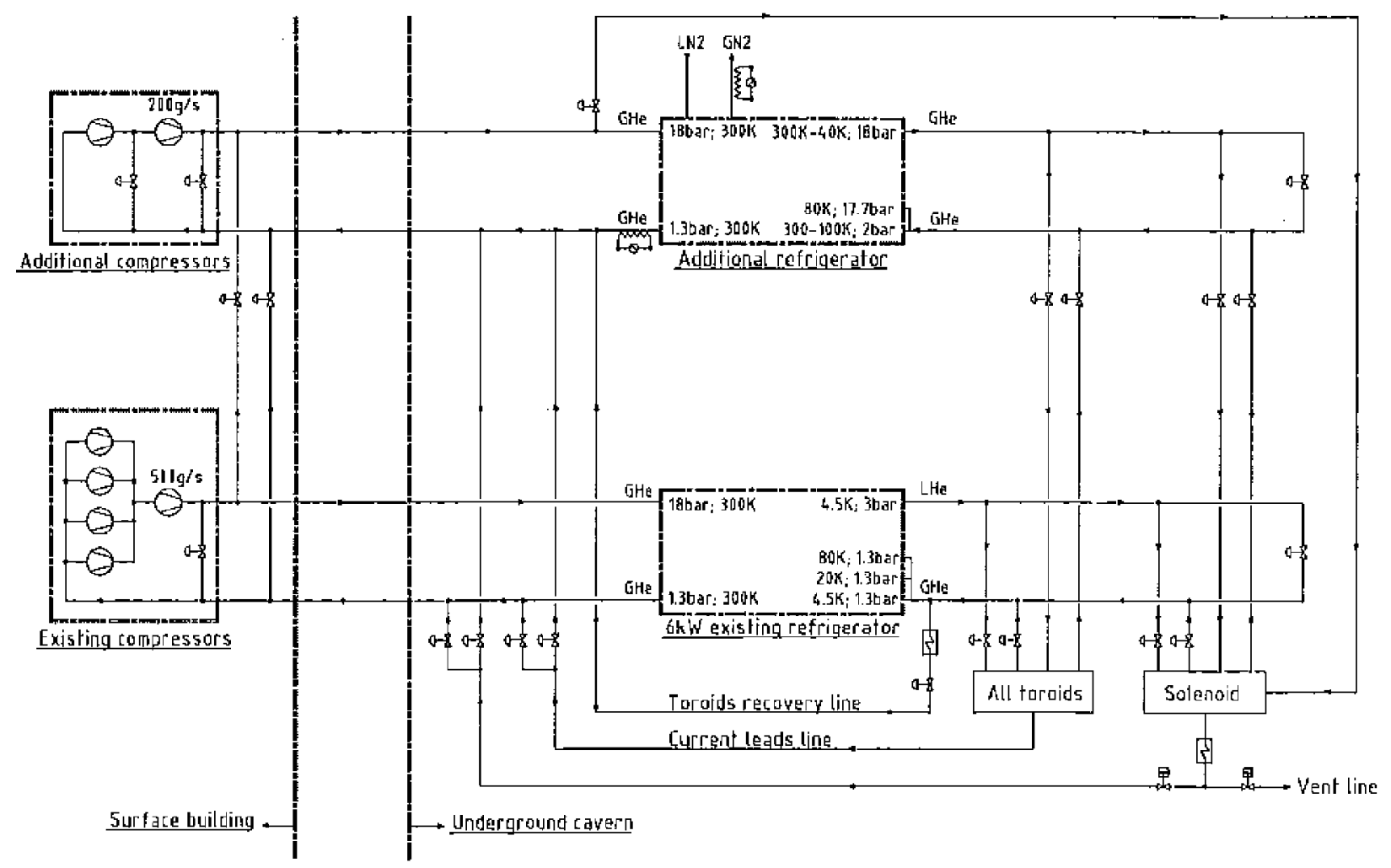

Fig.2: Helium distribution system for the ATLAS magnets

consisting of the BT, the two ECTs and the CS were regrouped into two sub-systems: one for all toroids and one for the CS. Furthermore, considering from a cryogenic point of view all toroids as a single cold mass minimizes the required number of transfor lines. Finally, the adopted design will allow the re-use without modifications of an existing $6 \mathrm{~kW}$ refrigerator that will he available by 2002 . The overall results are an important cost saving, an increase of the system reliability and a considerable improvement of the operational easiness.

\section{ACKNOWLEDGEMENT}

Special thanks to U. Wagner for his very valuable advices in thermodynamic process simulations.

\section{REFERENCES}

[1] ATLAS Technical Ptoposal, Decetrber 1994, ISBN 92-9083-067-0

[2] ATLAS Magnet System: Technical Design Repan, April 1997.

[3] H. Gtuehagen, "L'Air Liquide $6 \mathrm{~kW}$ Cyoplant for covering ATLAS Detector Cooling Needs", Diplona Thesis, CERNNTNU, April 1998
[4] F. Haug, J-P. Dauvergne, O. Passardi, D. Cragg, C. Curé, P. Pailler, C. Mayri, A. Yamamoto, "Refrigeration System for the ATLAS Experimert", $16^{\text {th }}$ International Cryogenic Lingineering Conference, Kitakyushu, Japan (1996), Pp. 111-114

[5] C. Mayri, C Curć, R. Duthi], D. Cragg, F, Haug, G. Passatdi, "Batrel Toroid Cryogenic System for the ATLAS Delector", $16^{\text {t. }}$ International Cryogenic Engineering Conference, Kitakyushu, Japan (1996), pp, 123-126

[6] D. Cragg, "The Cryogenic System of the ATLAS Experiment End Cap Toroids", $16^{\text {th }}$ Intemational Cryogenic Engineering Conference, Kitakyushu, Japas (1996), pp. 1 l5-1 l8

[7] K. Tanaka, A. Yamanoto, Y. Doi, Y. Makida, T. Haruyana, T. Kondo, "Cryogenic Design of the ATLAS Thin Superconducting Solenoid Magnet", $16^{\text {th }}$ International Cryogenic Engineering Conference, Kitakyushu, Japan (1996), pp. 119-122

[8] Yu,Y, Filippov, "Characteristies of Horizontal two-phase he]iun flows-Patt I: Flow Pattens and Void Fraction", Cryogenics, wol. 39 (1999), Pp. $59-68$

19] Yu.P. Filippov, "Chancteristics of Horizontal two-phase helium flows-Part II: Prossure Drop and Transient Heat Transfur", Cryogenics, vol. 39 (1999), pp. 69-75

[10] Y. Doi, A. Yamanoto, T. IIaniyama, Y. Makida, K. 'lanaka, H. Yamaoka, T. Kondo, "Ptoximity Cryogenic System for tho ATLAS Ceniral Solenoid (revision 1)", KEK, Japan, April 1999 